\title{
Monte Carlo Simulation of Surface-Charging Phenomena on Insulators Prior to Flashover in Vacuum
}

\author{
Kai-Kun Yu, Guan-Jun Zhang, Member, IEEE, Nan Zheng, Yevgeny Raitses, and Nathaniel J. Fisch
}

\begin{abstract}
Before flashover across an insulator under high electric field in vacuum, there are charging phenomena occurring on the insulator surface, which significantly affect the developing process of flashover. Based on the secondary-electron-emissionavalanche model and by using the Monte Carlo method, a 2-D analysis of surface charge density on cylindrical and conical insulators prior to flashover in vacuum has been performed under unipolar voltage. Different materials are employed, i.e., alumina ceramic, PTFE, PMMA, and PI. The influences of materials, voltage amplitudes, and coning angles on charge distribution are investigated. The results reveal that negative charges exist in a small surface region near the cathode, while the surface charges positive in a larger region away from the cathode. With increasing applied voltage, both the negative charge density and region decrease, and even vanish, whereas both the positive charge density and region increase, and the peaks of both regions move toward the cathode. For the conical insulator with a negative angle, the positive charge density is greater than that with a positive angle, and the simulation describes well experimental data relating the coning angle, the surface charge, and the flashover voltage.
\end{abstract}

Index Terms-Electron emission, flashover, Monte Carlo method, surface charging, vacuum insulation.

\section{INTRODUCTION}

W HILE BRIDGING a solid insulating material between energized electrodes in vacuum, surface-discharge phenomena often occur across the insulator, and its onset flashover voltage is much lower than the breakdown voltage of either the vacuum gap or the insulator with the same length. Since the phenomena greatly restrict the overall performance of a vacuum-dielectric system, it has been paid much attention for more than half a century [1]. Generally, a flashover event is considered as a process that begins with the electron emission

Manuscript received September 25, 2008; revised January 4, 2009. First published April 3, 2009; current version published May 8, 2009. This work was supported in part by the National Natural Science Foundation of China under Grants 50577054 and 50777051 and in part by the Program for New Century Excellent Talents in University by the Chinese Ministry of Education under Grant NCET-04-0943.

K.-K. Yu and N. Zheng are with the State Key Laboratory of Electrical Insulation and Power Equipment, School of Electrical Engineering, Xi'an Jiaotong University, Xi'an 710049, China (e-mail: yukkun@mail.xjtu.edu.cn).

G.-J. Zhang is with the State Key Laboratory of Electrical Insulation and Power Equipment, School of Electrical Engineering, Xi' an Jiaotong University, Xi'an 710049, China, and also with Beijing Key Laboratory of High Voltage and EMC, North China Electric Power University, Beijing 102206, China (e-mail: gjzhang@mail.xjtu.edu.cn; displasma@gmail.com).

Y. Raitses and N. J. Fisch are with Princeton Plasma Physics Laboratory, Princeton University, Princeton, NJ 08543 USA.

Color versions of one or more of the figures in this paper are available online at http://ieeexplore.ieee.org.

Digital Object Identifier 10.1109/TPS.2009.2015450 from the cathode triple junction (CTJ, the proximity of metal cathode, insulator, and vacuum), giving rise to the secondaryelectron-emission (SEE) avalanche (SEEA) due to the impact of emitted electrons on insulator surface, and ends with the breakdown occurring in desorbed-surface gas layer [2].

During the developing process of flashover, the insulator surface is charged due to the SEE phenomena, which greatly distort the local electric field and affect the flashover development [3], [4]. Therefore, it is needed to know the surface-charging behavior for deeply understanding the discharge and flashover mechanisms. However, up to now, it is still very difficult to measure the actual surface-charge distribution while applying voltage. Traditionally, the surface charges can be measured with an electrostatic probe after removing applied voltage [5]-[7]. Limited by its measurement principle, the result cannot indicate the real charge distribution prior to flashover with voltage application. Based on the Pockels effect, Yamano et al. [8] first reported the real-time surface-charge distribution on PET film with rod-plane electrode system while applying ac voltage in vacuum. This method is feasible for thin materials under quasi-steady voltage excitations (e.g., dc and power frequency ac). However, for common dielectrics, with other electrode configuration and under transient voltages, it is difficult to perform the measurement.

Based on the charge-simulation method, Pillai and Hackam [9] and Enloe [10] calculated the surface discrete charges on insulator to describe its charging phenomena. Surface charging during the flashover event is a dynamic and stochastic process. Therefore, based on the Monte Carlo method, De Tourreil and Srivastava [11] and Yamamoto et al. [12] performed the kinetic simulation with some relatively simplified assumptions concerning the electron emission.

In this paper, on the basis of SEEA model, a 2-D Monte Carlo code is used to simulate the charging with some more reasonable conditions. Different insulating materials with cylindrical or conical shapes are employed. The simulation results are useful for correlating the surface charging and final flashover characteristics in vacuum.

\section{TheOrETICAL ANALYSis AND Electrode CONFIGURATION}

During the developing process of surface flashover in vacuum, energetic electrons interact with the insulator surface, leading to the absorption or backscattering of incident electrons or emission of secondary electrons. The SEE rate $\delta$ is a function 
of the kinetic energy $E_{i}$ of electrons impinging upon the insulator surface with different incident angle $\phi$. Synthetically considering the descriptions of Burke [13] and Rodney and Vaughan [14], here, a comprehensive analytical expression is proposed to describe the SEE yield $\delta / \delta_{m}$ of insulating materials as follows. Compared with current formula, this description is more fitted to the experimental results reported [15]-[17]

$\delta\left(E_{i}, \phi\right) / \delta_{m}=1.526\left(1+k_{s} \phi^{2} / 2 \pi\right)\left(1-\exp \left(-z^{1.725}\right)\right) / z^{0.725}$

with

$$
z=1.284 E_{i} / E_{m}
$$

where $k_{s}$ is a smoothness factor of material surface ranged from 0 to 2 (e.g., 0 for textured carbon, 1.5-2 for a polished or crystalline surface, and 1 for usual surface); in this paper, all the materials used are assumed with the usual surface, and $k_{s}$ is selected as one, and $\phi$ is the incident angle to the normal direction.

Note that there are elastic and inelastic backscatterings of incident electrons in SEE, and while the energy of impact electron is below $50 \mathrm{eV}$, the backscattering of incident electrons has a considerable proportion. There are no sufficient data to precisely describe the contribution of backscattering electrons for dielectric materials. Some measurements suggest that backscattering electrons are responsible for local minimum and maximum on the SEE curves of dielectric materials in the low-energy range [18], [19]. This complex behavior is not addressed in this paper, where the backscattering proportion $\eta$ is taken into account in order to distinguish the "true" secondary electrons by using the following expression [13]:

$$
\eta\left(E_{i}\right)=0.115\left(E_{i} / 1000\right)^{-0.223} .
$$

The backscattering proportion $\eta$ can greatly affect the simulation result of sample surface charge density, particularly near the cathode where the electron energy is low, and in this paper, it is considered.

Four kinds of commonly used materials are employed in the simulation, i.e., alumina ceramic $\left(\mathrm{Al}_{2} \mathrm{O}_{3}\right)$, polytetrafluoroethylene (PTFE), polymethyl methacrylate (PMMA), and polyimide (PI), and their SEE curves with the incident electron angle $\phi=0$ are shown in Fig. 1 according to (1). The characteristic SEE values of these materials are listed in Table I [12], [13], [15]-[17].

The energy $E_{o}$ of emitted secondary electrons is somewhat difficult to be definitely determined. Based on experimental measurements of different materials (metals and insulators), Von Seggern [20] gave an approximately analytical description of $E_{o}$ as follows, which is valid for most insulation surface:

$$
g\left(E_{o}\right)=\sum_{i=1}^{2} a_{i} \exp \left(-b_{i} E_{o}\right)
$$

Here, with $a_{1}=0.476, a_{2}=-0.476, b_{1}=0.4$, and $b_{2}=2.5$. The probability distribution of secondary electrons with different energy $E_{o}$ is shown in Fig. 2. The energy of most electrons is of a few electronvolts.

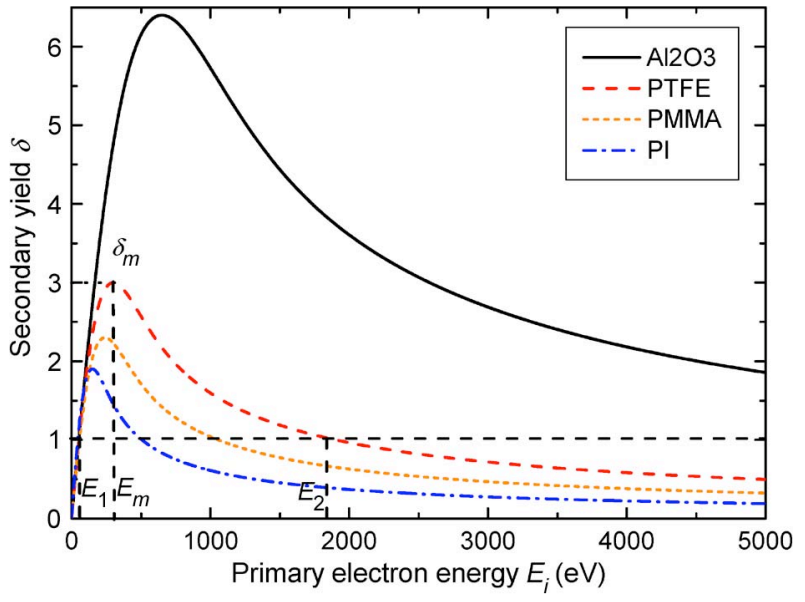

Fig. 1. SEE yield curve of different insulating materials.

TABLE I

SEE CHARACTERISTIC VALUES FOR DIFFERENT MATERIALS

\begin{tabular}{|c|c|c|c|c|}
\hline Materials & $\delta_{m}$ & $E_{m}$ & $E_{1}$ & $E_{2}$ \\
\hline $\mathrm{Al}_{2} \mathrm{O}_{3}$ & 6.4 & 650 & 52 & 11800 \\
\hline $\mathrm{PTFE}$ & 3.0 & 300 & 50 & 1850 \\
\hline PMMA & 2.3 & 240 & 56 & 1062 \\
\hline PI & 1.9 & 150 & 43 & 517 \\
\hline
\end{tabular}

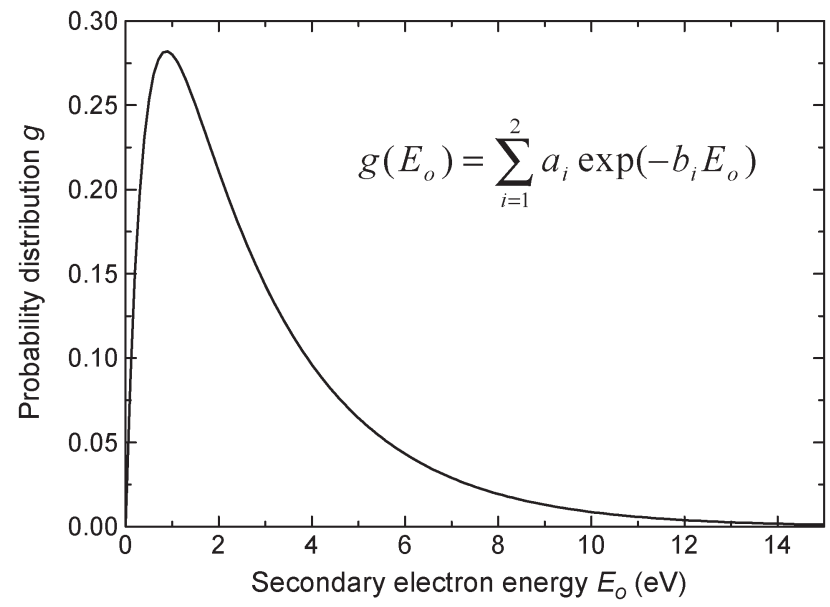

Fig. 2. Secondary-electron energy distribution.

Parallel flat electrodes with cylindrical or conical insulator are the commonly used electrode-sample configuration, as shown in Fig. 3. The stainless steel is employed as the electrode material. For the cylindrical insulator, it is with the radius of $10 \mathrm{~mm}$ and the height of $10 \mathrm{~mm}$, and for conical insulator, the radius of its narrow end is $10 \mathrm{~mm}$, and the other end is dependent on the coning angle $\theta$. These scales are used in the simulation. The applied voltage is considered as unipolar voltage in the simulation.

\section{Monte Carlo Simulation of SurfaCE-CHARgING PHENOMENA}

The Monte Carlo random-walk process is employed to simulate the charging phenomena, as follows.

Step 1) While the voltage is applied across the insulator, primary electrons are emitted from the CTJ, and 

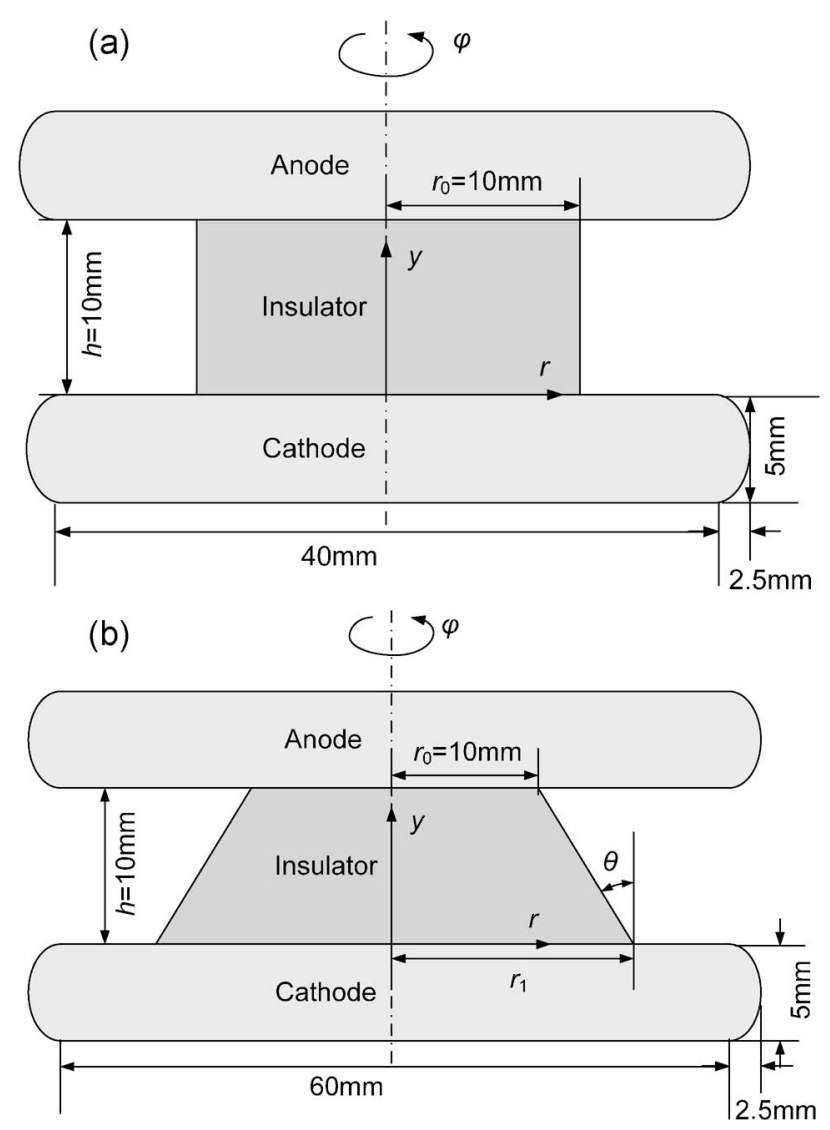

Fig. 3. Schematic of electrode-sample configuration for simulation (a) Cylindrical insulator. (b) Conical insulator.

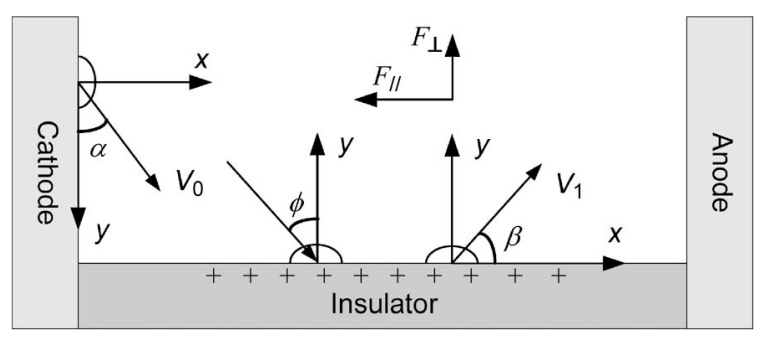

Fig. 4. Emission direction of primary and secondary electrons.

they are accelerated under the applied field. It is assumed that they are emitted as a bundle [11], [12]. Their emitted angle $\alpha$ is considered to obey a cosinelaw distribution about the normal axis perpendicular to the cathode [21], as shown in Fig. 4. Here, the charges' imaged in the electrode are not taken into account, because in comparison with the applied electric field, the image force due to image charges can be neglected.

Step 2) Some of the emitted electrons will hit the insulator surface with the incident angle $\phi$ due to their cosinelaw movement distribution, then secondary electrons may be emitted according to the SEE yield curve as shown in Fig. 1 fitted by (1), and (3) is considered to clarify the influence of electron backscattering. While $\delta>1$, secondary electrons are emitted, and the surface is positively charged, and otherwise, the surface acquires negative charges. The energy $E_{0}$ distribution of the secondary electrons is as shown in Fig. 2 fitted by (4). Previous studies usually simplified the emitted electrons to be monoenergetic [11], [12]. The emission direction $\beta$ of secondary electrons also obeys the cosine-law distribution about the insulator surface [21], as shown in Fig. 4.

Step 3) The secondary electrons are also accelerated by the electric field while traveling toward the anode in vacuum. Some of the secondary electrons will hit the insulator surface and may induce new secondary electrons before they arrive at the anode. After all the electrons reach the anode, the surface-charge distribution on the sample and also the electric field induced by the surface charges is calculated at once, then another bundle of electrons is emitted from the CTJ. In this process, the changed surface charge and electric field distribution is taken into account. The earlier steps are repeated until all electron bundles arrive at the anode.

It is assumed that the primary field-enhanced electrons are emitted from the cathode surface in a range of $0-10 \mu \mathrm{m}$ near the CTJ, the numbers of electrons in a bundle are $1 \times 10^{6} / \mathrm{cm}$, and 5000 bundles of electrons are emitted [12]. The energy of primary electrons emitted from the cathode is dependent on the cathode material and is approximately equal to its Fermi energy level $E_{F}$. For stainless-steel cathode, $E_{F}$ is $11.1 \mathrm{eV}$ in our simulations [21]. In the horizontal direction, it is considered that the movement of both primary and secondary electrons is dependent on the applied electric field $F_{/ /}=U / d$. In the vertical direction, their movement is dependent on the surfacecharge electric field $F_{\perp}=\sigma /\left(2 \varepsilon_{0}\right)$, which is greatly determined by the surface charges accumulated, and its initial value is zero while the first primary electrons are emitted. Although a negligibly small initial surface charge is a valid approximation for $\mathrm{Al}_{2} \mathrm{O}_{3}$ or for the organic dielectrics considered in this paper, for ferroelectrics, the influence of the initial surface charge due to polarization of the material is not negligible [22]. In fact, in ferroelectrics, the surface charge has been shown to influence the parameters of surface flashover, and the polarization of the ferroelectric material affects the first crossover energy of SEE. Here, the final energy and incident angle of each electron while hitting on the insulator surface is determined by the $F_{/ /}$and $F_{\perp}$. The effect of charge image force from the cathode and insulator is small enough to be neglected. The SEE properties of each insulator surface are assumed to remain stable, as in (1)-(3), and Fig. 1 while charging builds up, and to be homogeneous over the entire surface.

\section{RESUltS AND Discussion}

\section{A. Influence of Applied Voltage Amplitude on Surface-Charge Distribution}

Fig. 5 shows the charge distribution on cylindrical PMMA insulator under 10,20,30,40, and $50 \mathrm{kV}$, respectively.

From Fig. 5, it is shown that there are negative charges exist in a small region near the cathode and, then, turn to positive 


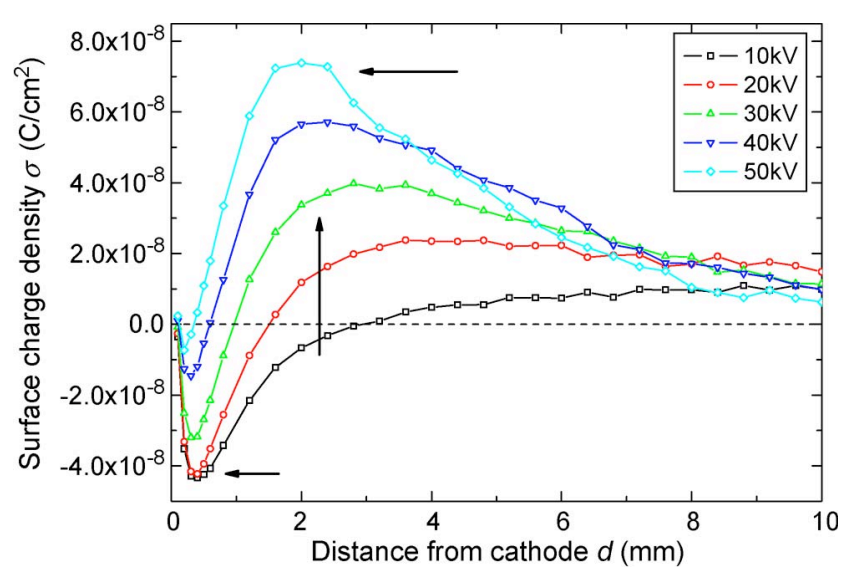

Fig. 5. Surface-charge distribution on cylindrical PMMA insulator under different voltage steps.

charges in a big region away from the cathode. While increasing the applied voltage, the density and area of negative charges decrease, but the density and area of positive charges increase, and both the peak of negative- and positive-charge regions move toward the cathode. This is because, while primary electrons just emit from the CTJ under a lower voltage, these electrons gain relatively lower energy under a lower applied electric field, and some of them with a small emissive angle $\alpha$ just transport over a short distance, then hit the insulator surface with the energy lower than $E_{1}$, thus resulting in negative-charging phenomena near the cathode. Obviously, for primary electrons with bigger $\alpha$, it is difficult for them to hit on the insulator unless the insulator surface is highly charged and the vertical electric field $F_{\perp}$ is high enough. For the area near to the anode, due to the high-energy gain of some electrons across a long transport distance under applied voltage, their energies are close to and even beyond another critical value $E_{2}$, leading to the relatively lower positive charge density. Moreover, for the area between the two electrodes, the energy of electrons corresponds to the curve region with a high SEE coefficient in Fig. 1, thus resulting in the higher positive-charging phenomena. Similarly, with the increase of applied voltage, the emitted electrons will gain higher energy from the applied electric field, thus resulting in that both the peak locations of negative and positive charges move toward the cathode. Both the density and area of positive charges increase, but both the density and area of negative charges decrease, as shown in Fig. 5.

In Fig. 5, while the applied voltage is $10 \mathrm{kV}$, the maximal values of the negative- and positive-charge densities are $\sim-4.34 \times 10^{-8}$ and $1.22 \times 10^{-8} \mathrm{C} / \mathrm{cm}^{2}$ and located at $\sim 0.4$ and $9.1 \mathrm{~mm}$ away from the cathode, respectively. However, while the voltage is $50 \mathrm{kV}$, the maximal values of the negativeand positive-charge densities are up to $\sim-7.27 \times 10^{-9}$ and $7.50 \times 10^{-8} \mathrm{C} / \mathrm{cm}^{2}$ and located at $\sim 0.2$ and $2 \mathrm{~mm}$ away from the cathode, respectively. Jaitly and Sudarshan [5] performed the flashover experiment across PMMA in vacuum (with PMMA length of $20 \mathrm{~mm}$ and dc voltage application), and they measured the surface-charge distribution between two electrodes via an electrostatic probe after flashover occurred. Moreover, the distribution tendency of the surface charge is nearly consistent with Fig. 5, particularly in the middle of two
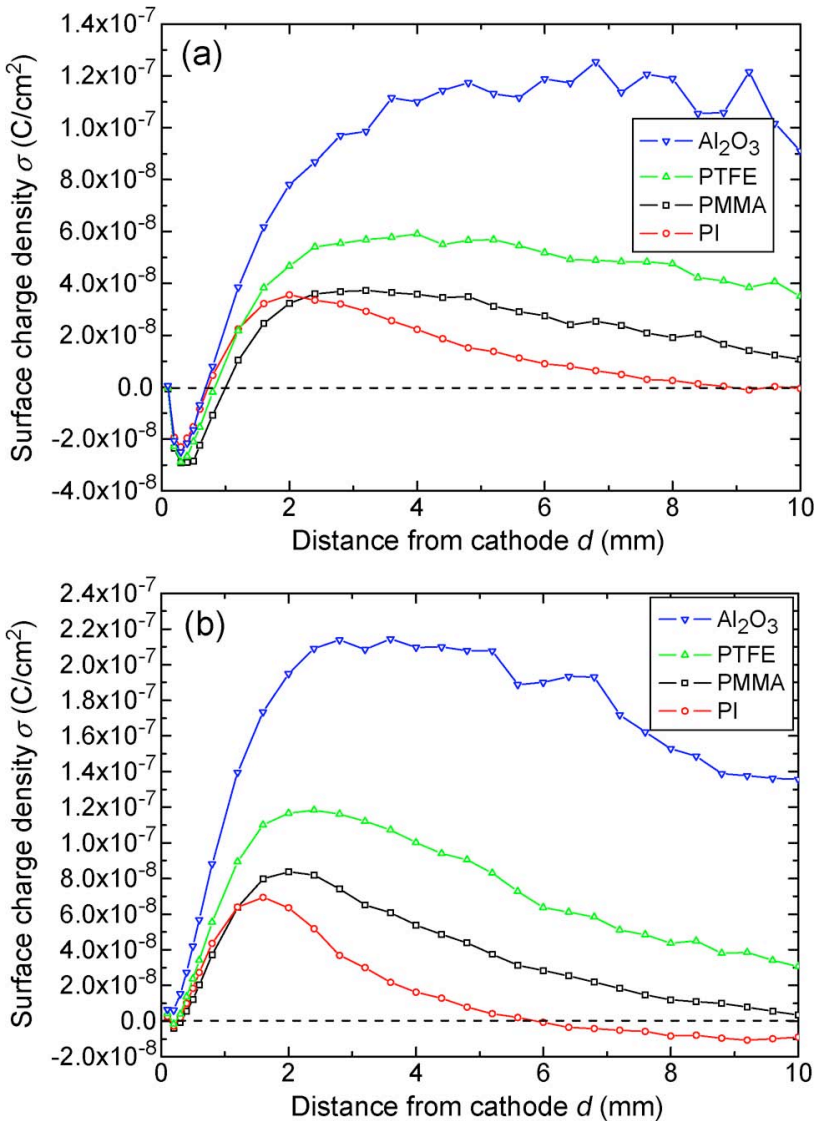

Fig. 6. Surface-charge distribution on different cylindrical insulators under different voltage steps. (a) Under $30 \mathrm{kV}$. (b) Under $50 \mathrm{kV}$.

electrodes. They did not give the charge distribution very close to the cathode and anode, because this kind of probe could not be located too near to the electrodes. Positive charges with high density near the cathode will result in the enhancement of local electric field and, henceforth, the occurrence of flashover phenomena.

\section{B. Surface-Charge Distribution of Different Materials}

Fig. 6 shows the charge distribution for cylindrical insulator with four different materials, and the applied voltage is 30 and $50 \mathrm{kV}$, respectively. It is shown that the charge distribution are various, with different materials and voltage amplitudes.

Compared with Fig. 1 and Table I, we can notice that, for PI with the smallest $\delta_{m}$ and the narrowest interval between $E_{1}$ and $E_{2}$, its surface positive charge density and region is the smallest among the four kinds of materials. On the contrary, the charge density of alumina ceramic is the largest, then PTFE and then PMMA. It is concluded that surface charge density is closely related with the SEE yield curve, and the higher the SEE rate, the larger the surface positive charge density. Under the applied voltage as high as $50 \mathrm{kV}$, the emitted electrons can gain higher energy from the applied electric field; the negativecharge region near to the cathode nearly disappears and even turns positive, as shown in Fig. 6(b).

Sudarshan et al. [23] first reported that, while coating alumina ceramics with $\mathrm{Cu}_{2} \mathrm{O}$ or $\mathrm{Cr}_{2} \mathrm{O}_{3}$ layer with low SEE coefficient, their flashover voltages are significantly improved, 
which indicates that SEE characteristics greatly affect the surface-charging phenomena and, thus, the flashover voltage of insulator. The authors conducted the flashover experiments of alumina and PTFE in vacuum and found that, under the same experimental conditions (with vacuum level of $4 \times 10^{-4} \mathrm{~Pa}$, gap spacing of $5 \mathrm{~mm}$, and dc voltage application), the flashover voltage of PTFE $(\sim 55 \mathrm{kV})$ is much higher than that of alumina ceramic $(\sim 45 \mathrm{kV})$ [24], which agrees with their surfacecharging differences shown in Fig. 6. Remarkably, we noticed that, after a flashover event, the surface charge density of PTFE is much higher than that of alumina. The possible reason is that the surface resistivity of PTFE is relatively higher than that of alumina, so the surface charges on PTFE leak more slowly than that on alumina after flashover shot and, thus, displays higher residual charges.

Due to the similar SEE curve of different materials while $E_{i}<E_{1}$ in Fig. 1, the peak position of negative charges is similar for different materials in Fig. 6. However, the peak position of positive-charge region for different materials is quite various because of the difference on their SEE curve while $E_{i}>E_{1}$, and the peak location and density is closely related to their different $E_{m}$ and $\delta_{m}$ as shown in Fig. 1. Under a higher applied voltage, the emitted electrons can gain higher energy from the applied electric field; their energy is easily greater than $E_{2}$ after accelerating across the field, and so, the area near to the anode corresponds to a lower SEE rate $\delta$, thus as near to the anode, the positive charge density decreases. For PI, the local charges near to the anode even turn negative under $50 \mathrm{kV}$.

\section{Influence of Coning Angle on Surface-Charge Distribution}

Fig. 7 shows the charge density on the conical PMMA and alumina insulator while changing its coning angle under $20 \mathrm{kV}$, respectively. Due to their different SEE yield, some differences are observed.

When the coning angle is negative, the surface positive charge density is significantly higher than when it is positive. This is because, when $\theta<0^{\circ}$, the electrons emitted from CTJ easily impact on the insulator surface along the direction of applied electric field, thus easily leading to the SEE phenomena. Based on the simulation results, when $\theta>0^{\circ}$, the positive charge density is usually lower than that when $\theta<0^{\circ}$, and at the same time, the negative charge density is relatively higher. It is very interesting that the largest positive charge density corresponds to the angle of $-20^{\circ} \sim-30^{\circ}$; the smallest positive charge density corresponds to the angle of $>30^{\circ}$. Moreover, for the negative angle of $<-30^{\circ}$, the positive charge density turns smaller. For a negative angle of $<-30^{\circ}$, the free path of emitted electrons is relatively shorter, so it is difficult for them to gain enough energy from the electric field and to achieve the SEE phenomena. When the positive angle is above $45^{\circ}$, there are nearly no positive charges observed, and only negative charges exist near the cathode. The reason is that, when the positive angle is greater than a critical value, the probability of emitted electrons hitting the insulator surface is much decreased, and they will easily fly away to the anode directly. Therefore, in pulsed-power and particle accelerators, the insulator stacks with $45^{\circ}$ are typically employed [25].
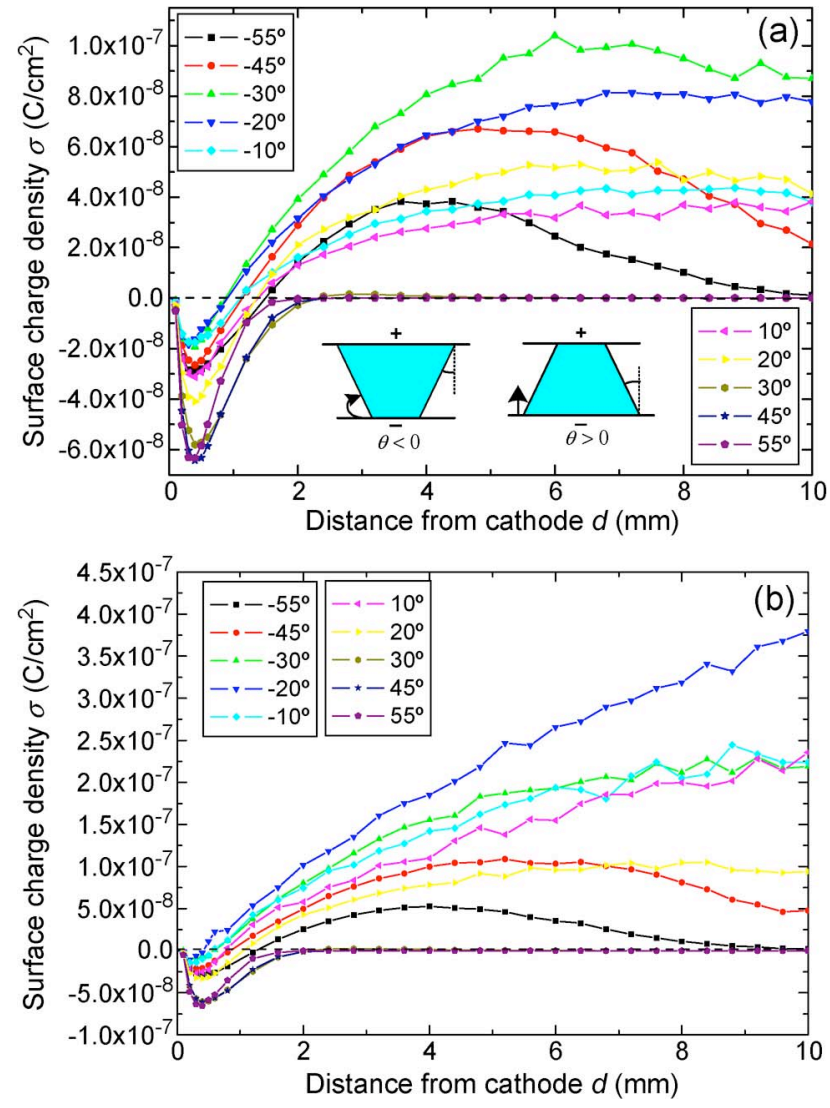

Fig. 7. Surface-charge distribution on conical insulators with different coning angles under $20 \mathrm{kV}$. (a) PMMA. (b) $\mathrm{Al}_{2} \mathrm{O}_{3}$.

Milton [26], Pillai and Hackam [27], and Yamamoto et al. [28] had reported the experimental flashover voltage of different materials with different coning angles. Although there is some discrepancies in their reported data, generally, the minimal flashover voltage appears at $-20^{\circ} \sim-30^{\circ}$, and the maximal voltage corresponds to the angle of $>30^{\circ}$, which highly agree with our simulation results as shown in Fig. 7. Note also that a transverse magnetic field affects the flashover characteristics by adjusting the movement angle of emitted electrons, and suitable magnetic field configuration can promote the surface withstanding strength [29].

\section{CONCLUSion}

A computer code using the Monte Carlo method is programmed to simulate the charge distribution on insulator surface between two flat electrodes prior to flashover in vacuum. The simulation results reveal that, under applied voltage across the insulator, a small surface region near the cathode is charged negatively, while a larger surface region away from the cathode charges positive. With increasing voltage, both the density and area of negative charges decrease, and may even vanish, but both the density and area of positive charges increase, and the peaks of both regions move toward the cathode. When the conical insulator's angle is $-20^{\circ}$ and $-30^{\circ}$, there is the largest positive charge density observed on its surface, and for angles above $30^{\circ}$, the positive charges gradually disappear. These results are consistent with flashover data reported both 
by us and by others. We are setting up the surface-charge measurement system, and the measurement results will be used to compare with these simulation results.

\section{REFERENCES}

[1] R. Hawley, "Solid insulators in vacuum-A review," Vacuum, vol. 18, no. 7, pp. 383-390, 1968.

[2] R. A. Anderson and J. P. Brainard, "Mechanism of pulsed surface flashover involving electron-stimulated desorption," J. Appl. Phys., vol. 51, no. 3, pp. 1414-1421, Mar. 1980.

[3] H. C. Miller, "Flashover of insulators in vacuum: Review of the phenomena and techniques to improved hold off voltage," IEEE Trans. Elect. Insul., vol. 28, no. 4, pp. 512-527, Aug. 1993.

[4] G. J. Zhang, Z. Yan, Y. S. Liu, K. Yasuoka, and S. Ishii, "Optical observation of preflashover phenomena from polytetrafluoroethylene in a planar concentric structure," J. Appl. Phys., vol. 93, no. 10, pp. 6405-6407, May 2003.

[5] N. C. Jaitly and T. S. Sudarshan, "In-situ insulator surface charge measurements in dielectric bridged vacuum gaps using an electrostatic probe," IEEE Trans. Elect. Insul., vol. 23, no. 2, pp. 261-273, Apr. 1988.

[6] Tumiran, M. Maeyama, H. Imada, S. Kobayashi, and Y. Saito, "Flashover from surface charge distribution on alumina insulators in vacuum," IEEE Trans. Dielectr. Elect. Insul., vol. 4, no. 4, pp. 400-406, Aug. 1997.

[7] L. J. Ding, Y. P. Tu, C. R. Li, J. C. Wang, and J. Z. Lv, "Measurement of the charge distribution on the insulator surface in vacuum," High Volt. Eng., vol. 29, no. 1, pp. 1-2, Apr. 2003. 23.

[8] Y. Yamano, M. Miyazaki, S. Kobayashi, and Y. Saito, "Measurement of 2-dimensional surface charge distributions under vacuum flashover events on insulators with sub-milli-second temporal resolution," in Proc. 22nd Int. Symp. Discharges Elect. Insul. Vacuum, Sep. 2006, pp. 152-155.

[9] A. S. Pillai and R. Hackam, "Modification of electric field at the solid insulator-vacuum interface arising from surface charges on the solid insulator," J. Appl. Phys., vol. 54, no. 3, pp. 1302-1313, Mar. 1983.

[10] C. L. Enloe, "The equilibrium charge distribution and electric field at a vacuum/dielectric interface," J. Appl. Phys., vol. 65, no. 9, pp. 3329-3334, May 1989.

[11] C. H. De Tourreil and K. D. Srivastava, "Mechanism of surface charging of high-voltage insulators in vacuum," IEEE Trans. Elect. Insul., vol. EI-8, no. 1, pp. 17-21, Mar. 1973.

[12] O. Yamamoto, T. Hara, H. Matsuura, and M. Hayashi, "Temporal behavior of surface charge accumulation in bridged vacuum gaps," IEEE Trans. Dielectr. Elect. Insul., vol. 2, no. 2, pp. 292-298, Apr. 1995.

[13] E. A. Burke, "Secondary emission from polymers," IEEE Trans. Nucl. Sci., vol. NS-27, no. 6, pp. 1760-1764, Dec. 1980.

[14] J. Rodney and M. Vaughan, "New formula for secondary emission yield," IEEE Trans. Electron Devices, vol. 36, no. 9, pp. 1963-1967, Sep. 1989.

[15] P. H. Dawson, "Secondary electron emission yields of some ceramics," J. Appl. Phys., vol. 37, no. 9, pp. 3644-3645, Apr. 1966.

[16] R. F. Willis and D. K. Skinner, "Secondary electron emission yield behaviour of polymers," Solid State Commun., vol. 13, no. 6, pp. 685-688, Sep. 1973.

[17] H. Seiler, "Secondary electron emission in the scanning electron microscope," J. Appl. Phys., vol. 54, no. 11, pp. r1-r18, Nov. 1983.

[18] S. A. Fridrikhov and A. R. Shul'man, "An investigation of the secondary electron emission by certain dielectrics art low primary electron energies," Sov. Phys., Solid State, vol. 1, pp. 1153-1159, Jun. 1960.

[19] A. Dunaevsky, Y. Raitses, and N. J. Fisch, "Secondary electron emission from dielectric materials of a Hall thruster with segmented electrodes," Phys. Plasmas, vol. 10, no. 6, pp. 2574-2577, Jun. 2003.

[20] H. Von Seggern, "Charging dynamics of dielectrics irradiated by low energy electrons," IEEE Trans. Nucl. Sci., vol. NS-32, no. 4, pp. 1503-1511, Aug. 1985.

[21] A. J. Dekker, Solid State Physics. New York: Prentice-Hall, 1957.

[22] A. Dunaevsky and N. J. Fisch, "Operation of ferroelectric plasma sources in a gas discharge mode," Phys. Plasmas, vol. 11, no. 5, pp. 2957-2963, May 2004.

[23] T. S. Sudarshan, J. D. Cross, and K. D. Srivastava, "Prebreakdown processes associated with surface flashover of solid insulators in vacuum," IEEE Trans. Electr. Insul., vol. EI-12, no. 3, pp. 200-208, Jun. 1977.

[24] K. K. Yu, N. Zheng, and G. J. Zhang, Surface Flashover Experimental Results. (unpublished).

[25] W. A. Stygar, J. A. Lott, T. C. Wagoner, V. Anaya, H. C. Harjes, H. C. Ives, Z. R. Wallace, G. R. Mowrer, R. W. Shoup, J. P. Corley, R. A. Anderson, G. E. Vogtlin, M. E. Savage, J. M. Elizondo, B. S. Stoltzfus, D. M. Andercyk, D. L. Fehl, T. F. Jaramillo,
D. L. Johnson, D. H. McDaniel, D. A. Muirhead, J. M. Radman, J. J. Ramirez, L. E. Ramirez, R. B. Spielman, K. W. Struve, D. E. Walsh, E. D. Walsh, and M. D. Walsh, "Improved design of a high-voltage vacuum-insulator interface," Phys. Rev. Spec. Top., Accel. Beams, vol. 8 , no. 5 , p. 050401 , May 2005.

[26] O. Milton, "Pulsed flashover of insulators in vacuum," IEEE Trans. Elect. Insul., vol. EI-7, no. 1, pp. 9-15, Mar. 1972.

[27] A. S. Pillai and R. Hackam, "Surface flashover of conical insulators in vacuum," J. Appl. Phys., vol. 56, no. 5, pp. 1374-1381, Sep. 1984.

[28] O. Yamamoto, T. Hara, T. Nakae, and M. Hayashi, "Effects of spark conditioning, insulator angle and length on surface flashover in vacuum," IEEE Trans. Elect. Insul., vol. 24, no. 6, pp. 991-994, Dec. 1989.

[29] A. Dunaevsky, Y. Raitses, and N. J. Fisch, "Ferroelectric cathodes in transverse magnetic fields," J. Appl. Phys., vol. 93, no. 6, pp. 3481-3485, Mar. 2003.

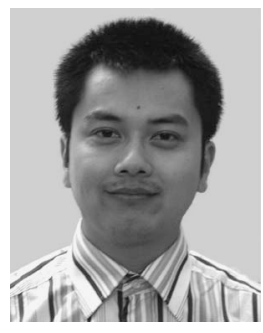

Kai-Kun Yu was born in Shaanxi Province, China, in 1981. He received the B.S. and M.S. degrees in electrical engineering from Xi' an Jiaotong University, Xi'an, China, in 2003 and 2006, respectively, where he is currently working toward the Ph.D. degree in the State Key Laboratory of Electrical Insulation and Power Equipment, School of Electrical Engineering.

His current research interests include pulsedsurface-flashover phenomena across the ceramic and composite organic materials.

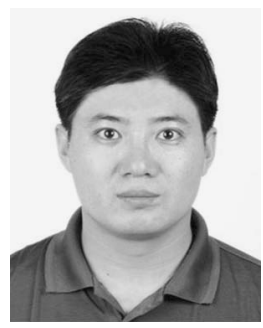

Guan-Jun Zhang (M'02) was born in Shandong Province, China, in October 1970. He received the B.S., M.S., and Ph.D. degrees in electrical engineering from Xi' an Jiaotong University (XJTU), Xi' an, China, in 1991, 1994, and 2001, respectively.

In July 1994, he was a Teacher Assistant with XJTU, where since June 2004, he has been a Full Professor with the State Key Laboratory of Electrical Insulation and Power Equipment, School of Electrical Engineering. From October 1998 to September 1999, he was a Visiting Researcher at Tokyo Institute of Technology, Tokyo, Japan, where he was engaged in surface electroluminescence and discharge phenomena of solid insulating materials. From August 2006 to February 2007, he was a Senior Visiting Fellow in Princeton Plasma Physics Laboratory, Princeton University, Princeton, NJ, where he was engaged in secondary-electron-emission characteristic and plasma simulation. From August to October 2008, he was a Japan Society for the Promotion of Science Fellow with Saitama University, Saitama, Japan, where he was engaged in flashover physics phenomena in vacuum. He is currently also an Adjunct Professor with the Beijing Key Laboratory of High Voltage and EMC, North China Electric Power University, Beijing, China. His current research interests include dielectric phenomena under high voltage, discharge physics and plasma technology, and condition maintenance of power equipment. He has published more than 100 journal articles and conference papers.

Dr. Zhang was the recipient of the 2008 Chatterton Young Investigator Award of the IEEE 23rd International Symposium on Discharges and Electrical Insulation in Vacuum, the 2006 Fok Ying Tong Research Award for University Young Teachers of China Ministry of Education (MOE), the 2003 National Top 100 Excellent Doctoral Dissertation Award of China MOE, and several other awards and prizes from the Chinese Government.



Nan Zheng was born in Shandong Province, China, in 1981. He received the B.S. degree in electrical engineering from Xi' an Jiaotong University, Xi'an, China, in 2005, where he is currently working toward the $\mathrm{Ph} . \mathrm{D}$. degree in the State Key Laboratory of Electrical Insulation and Power Equipment, School of Electrical Engineering.

His current research interests include surfacedischarge phenomena and surface-charging diagnostics across solid dielectrics. 
Yevgeny Raitses received the Ph.D. degree from the Technion-Israel Institute of Technology, Haifa, Israel.

He has previously held research positions with the Propulsion Physics Laboratory, Soreq NRC, Israel. Since 1998, he has been with Princeton Plasma Physics Laboratory, Princeton University, Princeton, NJ, where he is currently leading research on plasma thrusters and related plasma technologies. His current research is focused on plasma-wall interactions in gas discharges, physics and applications of low-temperature magnetized plasmas, and plasma diagnostics.

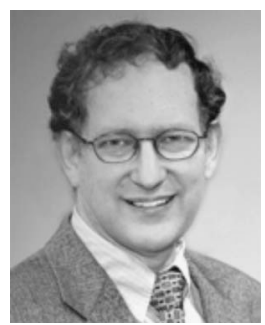

Nathaniel J. Fisch received the B.S., M.S., and $\mathrm{Ph} . \mathrm{D}$. degrees in electrical engineering and computer science from the Massachusetts Institute of Technology (MIT), Cambridge, in 1972, 1975, and 1978, respectively.

He is currently a Professor of astrophysical sciences and the Director of the Program in Plasma Physics with Princeton University, Princeton, NJ, where he is also an Associate Director for Academic Affairs, the Head of the Hall Thruster Laboratory, Princeton Plasma Physics Laboratory, and an Associated Faculty with the Department of Mechanical and Aerospace Engineering. $\mathrm{He}$ is best known for predicting new ways to drive electric current in hot magnetized plasma by means of electromagnetic waves. In addition to plasma thrusters and related plasma devices, his current research interests include plasma-based methods of generating extreme laser intensities and fusion concepts employing magnetically or inertially confined plasma.

Prof. Fisch was an MIT National Scholar. He was the recipient of the 2005 James Clerk Maxwell Prize of the American Physical Society, the Ernest Orlando Lawrence Award in 2004, the Department of Energy Bronze Medal for Outstanding Mentor in 2002, and the American Physical Society Award for Excellence in Plasma Physics in 1992. He is a Fellow of the American Physical Society and a Fellow of the NASA Institute for Advanced Concepts. He was the recipient of the Guggenheim Fellowship in 1985. In 1998, he was the Chair of the Division of Plasma Physics of the American Physical Society. 